\title{
Artrodese versus artroplastia da primeira articulação metatarsofalângica no tratamento do hallux rigidus - Estudo comparativo de pacientes selecionados apropriadamente*
}

\section{Arthrodesis versus Arthroplasty of the First Metatarsophalangeal Joint in the Treatment of Hallux Rigidus - A Comparative Study of Appropriately Selected Patients}

\author{
Marta Santos Silva ${ }^{1}$ Ricardo Rodrigues-Pinto ${ }^{1,2,3,4}$ (i) \\ 1 Departamento de Ortopedia, Centro Hospitalar do Porto, Hospital
de Santo António, Porto, Portugal
${ }^{2}$ Instituto de Ciências Biomédicas Abel Salazar, Porto, Portugal
${ }^{3}$ Trofa Saúde Hospital Privado de Alfena, Alfena, Portugal
${ }^{4}$ Trofa Saúde Hospital Privado Braga Centro, Braga, Portugal
${ }^{5}$ Centro Hospitalar Póvoa de Varzim/ Vila do Conde, Largo da
Misericórdia, Póvoa de Varzim, Portugal
}

Luís H. Barros ${ }^{1}$ Arnaldo Sousa ${ }^{1}$ José Muras $s^{3,4,5}$

Endereço para correspondência Ricardo Rodrigues-Pinto, PhD, Largo Prof. Abel Salazar, Porto, 4099-001, Portugal (e-mail: ric_pinto@hotmail.com).

Rev Bras Ortop 2020;55(1):40-47.

\section{Resumo}
Palavras-chave
- hallux rigidus
- artroplastia
- artrodese
- estudo comparativo
- artroplastia de substituição

Objetivo Historicamente, os resultados da artroplastia da primeira articulação metatarsofalângica (1MTP) eram relativamente ruins; no entanto, melhorias na compreensão da biomecânica normal do pé, nos materiais e no design dos implantes, tornam a artroplastia um tratamento aceitável em pacientes selecionados. O presente estudo pretendeu comparar os resultados clínicos e radiográficos da artrodese com os da artroplastia da 1MTP no tratamento de hallux rigidus e apresentar um racional para seleção de pacientes para artroplastia.

Métodos Um total de 36 pacientes (38 pés) com hallux rigidus operados (12 artrodeses e 26 artroplastias) foram prospectivamente incluídos. A dor foi avaliada com recurso à escala visual analógica (VAS) e o resultado funcional usando a escala American Orthopaedic Foot and Ankle Society Hallux Metatarsophalangeal-Interphalangeal (AOFAS-HMI). As complicações e os resultados radiográficos foram também registrados e a taxa de sobrevida calculada para os dois procedimentos.

Resultados Todos os pacientes referiram uma melhoria significativa na dor e nos resultados funcionais após a cirurgia. Os pacientes submetidos a artroplastia tiveram melhor resultado funcional na escala AOFAS-HMI $(89,7$ versus 65.7 pontos; $p<0.001) \mathrm{e}$ melhor alívio da dor (VAS 1,6 versus 3,9 pontos; $p=0,002$ ) quando comparados com os

\footnotetext{
Estudo conduzido no Departamento de Ortopedia, Centro Hospitalar do Porto, Hospital de Santo António, Porto, Portugal.
}

recebido

12 de Julho de 2018

aceito

04 de Dezembro de 2018
DOI https://doi.org/

$10.1055 / \mathrm{s}-0039-1700815$. ISSN $0102-3616$.
Copyright $(2020$ by Sociedade Brasileira License terms de Ortopedia e Traumatologia. Published by Thieme Revinter Publicações Ltda, Rio de Janeiro, Brazil 
doentes submetidos a artrodese. Registrou-se um caso de infecção no grupo da artroplastia e 2 casos de pseudoartrose no grupo da artrodese.

Conclusão A artrodese permite alívio da dor e resultados satisfatórios, mas altera a biomecânica da marcha. Tal como a artrodese, a artroplastia melhora a dor significativamente, sendo uma alternativa mais fisiológica para preservar a biomecânica do pé. Apesar dos dois tratamentos terem bons resultados clínicos, em pacientes selecionados, a artroplastia teve melhores resultados clínicos e menor taxa de revisão.

\section{Abstract}

\section{Keywords \\ - hallux rigidus \\ - arthroplasty \\ - arthrodesis \\ - comparative study \\ - joint replacement}

Objective Historical results of arthroplasty of the first metatarsophalangeal joint (1MTP) are relatively poor; however, improvements in the understanding of the normal foot biomechanics, implant materials and design currently make arthroplasty a reasonable option in appropriately selected patients. The present study aimed to compare the clinical and radiographic results of 1MTP arthrodesis and arthroplasty in the treatment of hallux rigidus and to present a rationale for patient selection for arthroplasty.

Methods A total of 36 patients (38 feet) with hallux rigidus submitted to surgery (12 arthrodesis and 26 arthroplasties) were prospectively included in the study. Pain was assessed using the visual analogue scale (VAS) and the functional status was assessed using the American Orthopedic Foot and Ankle Society Hallux MetatarsophalangealInterphalangeal (AOFAS-HMI) scale. Complications and radiographic results were also analyzed, and survival rates were calculated for both procedures.

Results All of the patients reported significant improvement in pain and functional status after surgery. Patients submitted to arthroplasty had better functional results on the AOFAS-HMI scale ( 89.7 versus 65.7 points; $p<0.001$ ) and better pain relief (VAS 1.6 versus 3.9 points; $p=0.002$ ) when compared with the group submitted to arthrodesis. There was one case of infection in the arthroplasty group and 2 cases of pseudarthrosis in the arthrodesis group.

Conclusion Arthrodesis provides pain relief and satisfactory results but alters the biomechanics of gait. Like arthrodesis, arthroplasty improves pain significantly, being a more physiological alternative to preserve the biomechanics of the foot. While the two surgical methods yielded good clinical results, selected patients submitted to arthroplasty had better clinical scores and lower revision rates.

\section{Introdução}

Hallux rigidus é uma doença degenerativa da primeira articulação metatarsofalângica (1MTP); sua prevalência é de 2,5\% em pessoas com $>50$ anos de idade e afeta principalmente mulheres $(2: 1) .^{1,2}$ A causa é considerada idiopática, embora quase dois terços dos pacientes tenham histórico familiar positivo e $79 \%$ apresentem acometimento bilateral. O hallux rigidus também é frequentemente associado a outras doenças, como hálux valgo interfalângico e metatarso aduto. ${ }^{1,3}$ Clinicamente, é caracterizado por dor, rigidez e limitação funcional, principalmente com dorsiflexão da 1MTP. Os achados físicos são a presença de uma proeminência dorsal acima da 1MTP correlacionada ao esporão ósseo dorsal à cabeça do metatarso, acompanhado por inflamação, aumento de volume, sensibilidade à palpação e diminuição da amplitude de movimento (ROM) da 1MTP. ${ }^{4}$

A classificação mais comum do hallux rigidus foi descrita pela primeira vez por Coughlin et al e é baseada em achados clínicos e radiográficos. ${ }^{5} \mathrm{O}$ tratamento não cirúrgico de casos brandos (estágios I e II) leva à melhora clínica e é composto por medicamentos anti-inflamatórios não esteroidais orais ou tópicos, injeção intra-articular de corticosteroides ou hialuronato de sódio, órteses de suporte, fisioterapia e modificações no estilo de vida. O tratamento cirúrgico é indicado quando o tratamento conservador é ineficaz e em estágios posteriores da doença. Consiste em debridamento articular e sinovectomia, queilectomia e osteotomia de descompressão nos estágios iniciais e hemiartroplastia, artroplastia total, artroplastia do tipo resurfacing e artrodese nos estágios avançados. ${ }^{3,6}$

A artrodese da articulação 1MTP foi descrita pela primeira vez por Broca em 1852 e ainda é considerado o tratamento padrão-ouro do hallux rigidus em estágio III e IV. Essa técnica diminui a dor e resultados de estudos em longo prazo mostraram trazer resultados favoráveis ao paciente. ${ }^{5,7,8} \mathrm{~A}$ artroplastia de 1MTP foi desenvolvida pela primeira vez na década de 1950 como uma alternativa à artrodese para preservação do movimento articular e da biomecânica normal do pé. Vários implantes diferentes foram desenvolvidos, mas os primeiros resultados foram decepcionantes. ${ }^{9,10}$ No entanto, novas melhorias no entendimento da biomecânica normal do pé, dos 
materiais e projetos de implante geraram melhores resultados e hoje formam uma alternativa razoável à fusão de $1 \mathrm{MTP} .{ }^{11-13}$

O objetivo do presente estudo foi comparar os resultados clínicos da artrodese e artroplastia de 1MTP no tratamento do hallux rigidus e justificar a seleção de pacientes para artroplastia.

\section{Material e Métodos}

\section{Delineamento Experimental e Participantes}

Entre 2007 e 2015, 38 pés de 36 pacientes com hallux rigidus em estágio III e IV foram submetidos a tratamento cirúrgico. Um total de 12 pacientes foi submetido a artrodese e 24 pacientes (26 pés) foram submetidos a artroplastia total de 1MTP. Os critérios de exclusão foram infecção/inflamação ativa, perda óssea grave ou destruição articular rápida, necrose avascular, instabilidade da articulação 1MTP, neuroartropatia de Charcot e intolerância/alergias a metais. Pacientes com desvio do eixo metatarsofalângico (hálux varo ou valgo), anomalias do arco do pé (pé plano ou cavo) ou retração do sistema calcâneo-plantar não foram selecionados para artroplastia e foram submetidos à artrodese. Apenas pacientes com período mínimo de acompanhamento de 2 anos foram incluídos no estudo.

$\mathrm{O}$ trabalho aqui apresentado foi aprovado pelo comitê de ética local e realizado de acordo com os padrões éticos estabelecidos pela Declaração de Helsinque de 1964 e suas alterações posteriores.

\section{Procedimentos Cirúrgicos e Cuidado Pós-operatório}

Todos os pacientes foram operados pelo mesmo cirurgião na mesma instituição. A cirurgia foi realizada sob anestesia geral ou peridural, com o paciente em decúbito dorsal na mesa cirúrgica. Um apoio foi colocado sob o quadril ipsilateral para girar internamente o membro inferior em um ângulo neutro; além disso, um torniquete foi colocado na coxa para exsanguinação. Todos os pacientes foram submetidos à antibioticoterapia profilática pré-operatória, que foi mantida nas primeiras 24 horas após a cirurgia.

A artrodese de 1MTP foi realizada com placa dorsal e parafusos (em sete pacientes) ou apenas com parafusos cruzados (em cinco pacientes), como já descrito. ${ }^{14-16}$

A mesma prótese (Metis, Newdeal SA; Integra Lifesciences ILS, Plainsboro Township, NJ, EUA) foi utilizada na artroplastia de 1MTP. Trata-se de uma prótese modular de titânio, não restritiva, com três componentes, não cimentada e revestida por hidroxapatita. Uma incisão medial do meio da falange ao meio do metatarso foi realizada para expor a primeira articulação metatarsofalângica. No preparo das superfícies articulares, osteófitos e cartilagens foram removidos e uma bunionectomia (quando necessária) foi realizada. Após a determinação do tamanho do componente metatársico, o metatarso foi seccionado com a guia de corte e o implante foi testado. É importante destacar que a secção do metatarso foi realizada em todos os pacientes com index plus (primeiro metatarso mais longo do que o segundo metatarso) para que os dois ossos apresentassem o mesmo comprimento (index plus minus). A falange foi, então, preparada com mandril e o implante de tamanho apropriado foi escolhido e testado. A amplitude de movimento e a frouxidão

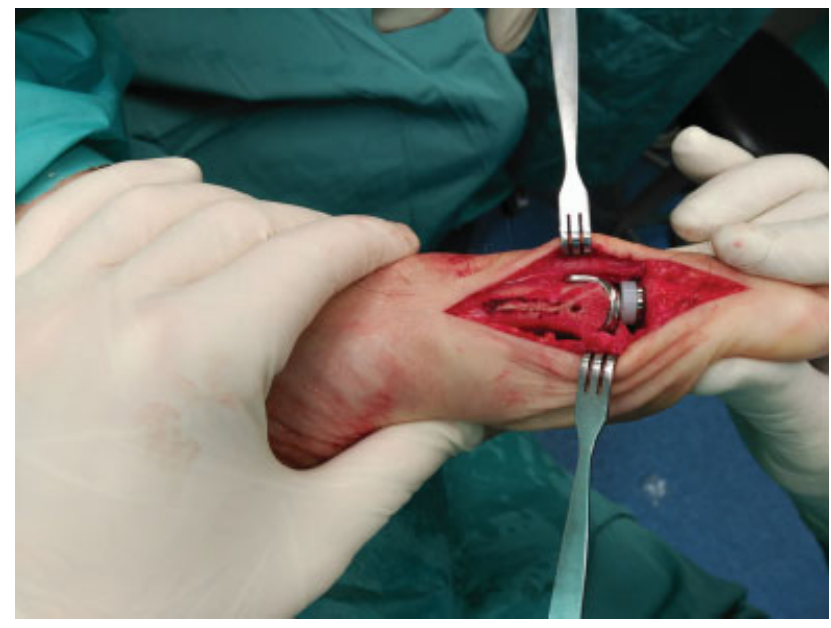

Fig. 1 Fotografia intraoperatória da artroplastia de 1MTP mostrando o encaixe correto dos implantes.

dos implantes testados foram verificadas para escolha da espessura do revestimento. A fluoroscopia foi utilizada para avaliar o alinhamento correto dos diferentes componentes. Os implantes finais de falange, metatarso e revestimento foram inseridos sob pressão (-Fig. 1). Um relatório preliminar desses resultados já foi publicado. ${ }^{17}$

Após a cirurgia, todos os pacientes foram submetidos à tromboprofilaxia. O material de sutura foi removido 15 dias após a cirurgia. No grupo submetido à artrodese, a sustentação parcial de peso começou em 2 semanas, e a sustentação total de peso foi iniciada em 6 semanas. No grupo submetido a artroplastia, a sustentação parcial de peso com sandália de Barouk começou em 2 semanas, e a sustentação total de peso em 6 semanas.

\section{Variáveis de Desfecho}

O tempo de acompanhamento, o tempo até a revisão, a idade do paciente à cirurgia e as complicações foram registradas. Os pacientes foram atendidos em 2 e 6 semanas, 3 e 6 meses e 1 vez ao ano. Os pacientes submetidos à artrodese receberam alta após o término do tratamento sem complicações, enquanto os pacientes submetidos a artroplastia foram atendidos uma vez ao ano para verificação de sinais clínicos ou radiográficos de complicações.

A avaliação funcional foi realizada com a pontuação American Orthopedic Foot and Ankle Society Hallux Metatarsophalangeal-Interphalangeal (AOFAS-HMI) no pré-operatório e na última consulta de acompanhamento. A escala AOFAS-HMI mede dor, função e alinhamento em uma escala de 1 a 100 pontos. A avaliação da dor foi realizada com a escala visual analógica (VAS) antes da cirurgia e na última consulta de acompanhamento. A pontuação VAS mede a dor em uma escala de 1 a 10 , em que 0 indica a ausência de dor e 10 se refere à pior dor que se possa imaginar.

Radiografias simples em incidência anteroposterior (AP) e em perfil foram obtidas para avaliação do alinhamento do implante e evidências de linhas radiotransparentes na última consulta de acompanhamento. A presença de uma linha radiotransparente com $>1 \mathrm{~mm}$ em qualquer uma das interfaces da prótese foi considerada indicativa do afrouxamento do implante. 
Tabela 1 Dados demográficos dos pacientes submetidos a artrodese e a artroplastia

\begin{tabular}{|l|l|l|l|}
\hline & $\begin{array}{l}\text { Artrodese } \\
(\boldsymbol{n}=\mathbf{1 2})\end{array}$ & $\begin{array}{l}\text { Artroplastia } \\
(\boldsymbol{n}=\mathbf{2 6})\end{array}$ & valor-p \\
\hline Sexo & $\begin{array}{l}10 \text { mulheres } \\
(83,3 \%)\end{array}$ & $\begin{array}{l}16 \text { mulheres } \\
(61,15 \%)\end{array}$ & 0,179 \\
& $\begin{array}{l}2 \text { homens } \\
(16,7 \%)\end{array}$ & $\begin{array}{l}10 \text { homens } \\
(38,46 \%)\end{array}$ & \\
\hline Idade (anos) & $57,6(50-70)$ & $63,5(59-83)$ & 0,005 \\
\hline $\begin{array}{l}\text { Acompanhamento } \\
\text { (meses) }\end{array}$ & $40,1(31-70)$ & $80,7(26-110)$ & $<0,001$ \\
\hline
\end{tabular}

\section{Análise Estatística}

O programa IBM SPSS Statistics for Windows, versão 23 (IBM Corp., Armonk, NY, EUA) foi utilizado para análise estatística. A estatística descritiva foi calculada. As médias de dados de distribuição normal foram determinadas. Os testes de quiquadrado, t e U de Mann-Whitney, dependendo da variável analisada, foram utilizados para comparação dos dois grupos. As curvas de sobrevida dos dois grupos de pacientes foram calculadas segundo o método de Kaplan-Meier com intervalo de confiança (IC) de $95 \%$ e tiveram a cirurgia de revisão como desfecho. 0 critério para determinação do significado estatístico foi estabelecido em $\mathrm{p}<0,05$ para todas as comparações.

\section{Resultados}

Houve predomínio de mulheres nos dois grupos (83,3\% no grupo submetido à artrodese e $61,15 \%$ no grupo submetido à artroplastia, $p<0,05)$. A idade média dos pacientes foi de 57,6 anos (de 50 a 70 anos) no grupo submetido à artrodese e de 64,3 anos (de 56 a 83 anos) no grupo da artroplastia $(p=0,005)$. O tempo médio de acompanhamento foi de 40,1 meses (de 31 a 70 meses) e de 80,7 meses (de 26 a 110 meses), respectivamente (-Tabela $\mathbf{1}$ ).

Os pacientes dos dois grupos apresentaram melhora nas pontuações clínicas. A pontuação AOFAS-HMI melhorou de uma média de 40,2 para 65,7 pontos no grupo submetido à artrodese $(p<0,001)$ e de uma média de 43,2 para 89,7 pontos $(p<0,001)$ no grupo da artroplastia. As pontuações AOFAS-HMI na última consulta de acompanhamento foram, porém, maiores no grupo submetido à artroplastia do que no grupo da artrodese $(p<0,001)$. As pontuações da VAS também melhoraram significativamente nos grupos de artrodese (de 7,8 a 3,9 pontos, $p<0,001$ ) e artroplastia (de 8,0 a 1,6 pontos, $p<0,001$ ). Os valores de VAS na última consulta de acompanhamento foram significativamente menores nos pacientes submetidos à artro-

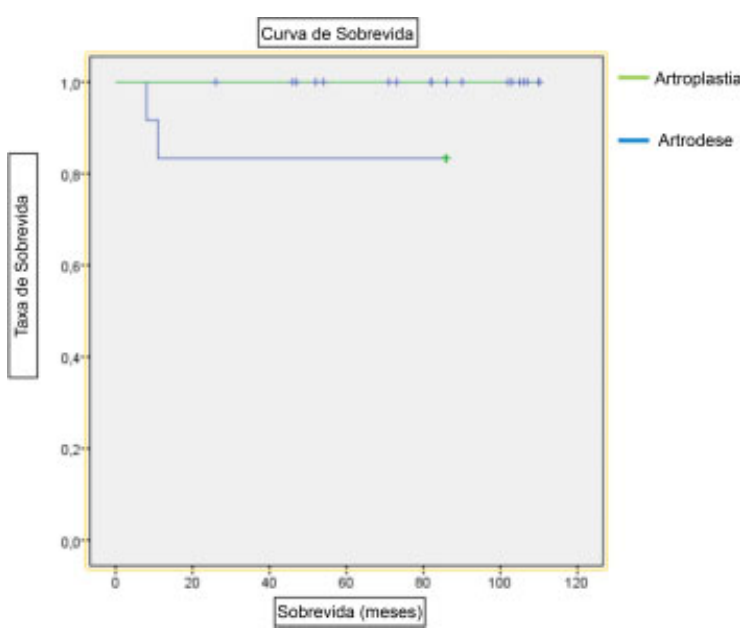

Fig. 2 Curvas de sobrevida da artroplastia e da artrodese.

plastia em comparação àqueles submetidos à artrodese (1,6 versus 3,9 pontos; $p=0,002$ ) (-Tabela 2 ).

Houve 3 complicações no grupo da artrodese: 1 (5,5\%) caso de metatarsalgia de transferência e $2(16,6 \%)$ casos de não consolidação, que foram submetidos a uma revisão de fusão com autoenxerto. Um caso de não consolidação ocorreu em uma fusão com parafusos cruzados, enquanto o outro foi observado em uma fusão com placa dorsal. No grupo submetido à artroplastia, houve um caso de infecção da ferida no período pós-operatório imediato, que foi tratado com antibióticos e cuidados específicos. Nenhuma outra complicação, como lesão neurovascular ou afrouxamento da prótese, foi registrada.

Tendo a cirurgia de revisão como desfecho, a taxa de sobrevida da prótese foi de $100 \%$, e a sobrevida da artrodese foi de $83 \%$ (-Fig. 2).

As-Figs. 3 e 4 ilustram os casos pré e pós-operatórios de artrodese e artroplastia. O Vídeo 1 mostra um paciente submetido à artroplastia bilateral.

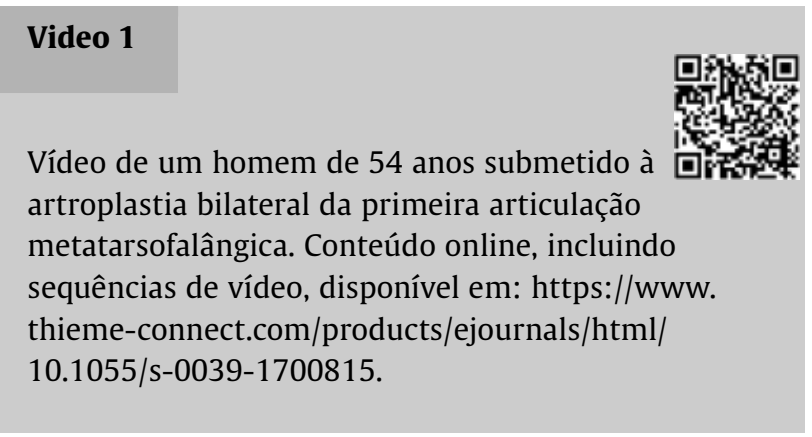

Tabela 2 Pontuações pré e pós-operatórias de AOFAS-HMI e VAS em pacientes submetidos à artrodese e à artroplastia

\begin{tabular}{|l|l|l|l|l|l|l|l|}
\hline \multirow{2}{*}{ PONTUAÇÕES } & \multicolumn{2}{|l|}{ Artrodese $(\boldsymbol{n}=12)$} & \multicolumn{2}{l|}{ Artroplastia $(\boldsymbol{n}=\mathbf{2 6})$} & \multirow{2}{*}{ valor-p* } \\
\cline { 2 - 8 } & Pré-operatório & Pós-operatório & valor-p & Pré-operatório & Pós-operatório & valor-p & \\
\hline AOFAS-HMI & $40,2(37-50)$ & $65,7(55-77)$ & $<0,001$ & $43,2(34,1-53,1)$ & $89,7(67-100)$ & $<0,001$ & $<0,001$ \\
\hline VAS & $7,8(5-10)$ & $3,9(0-6)$ & $<0,001$ & $8,0(5-10)$ & $1,6(0-3)$ & $<0,001$ & 0,002 \\
\hline
\end{tabular}

Abreviações: AOFAS-HMI, Pontuação American Orthopedic Foot and Ankle Society Hallux Metatarsophalangeal-Interphalangeal; VAS, escala visual analógica. *(comparação entre artrodese e artroplastia na última consulta de acompanhamento). 
44 Artrodese versus artroplastia da primeira articulação metatarsofalângica Silva et al.
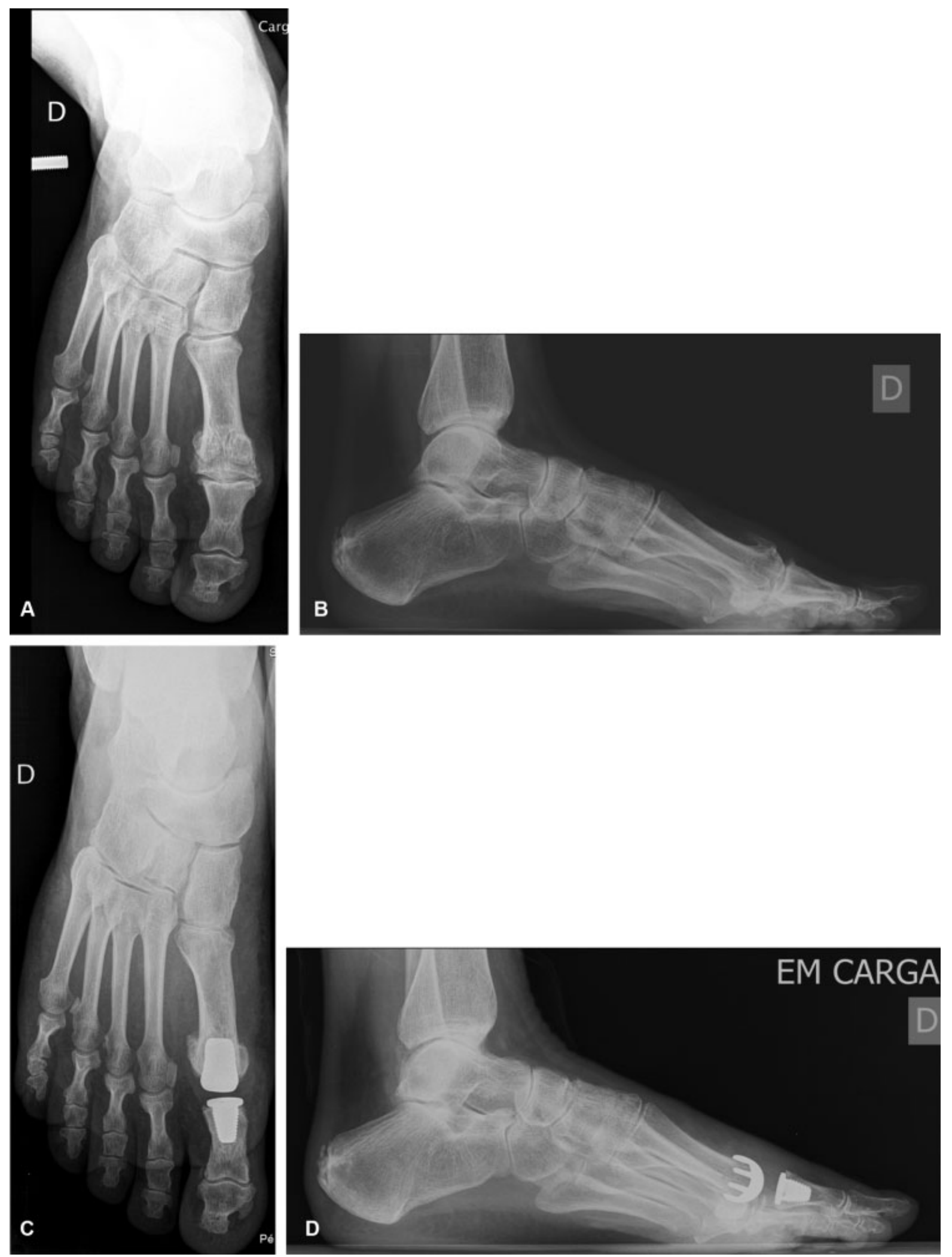

Fig. 3 Imagens radiográficas pré-e pós-operatórias de um homem de 62 anos de idade com hallux rigidus submetido à artrodese com parafusos cruzados. (A) Radiografia pré-operatória em incidência anteroposterior com o paciente em pé; (B) Radiografia pré-operatória em incidência em perfil com o paciente em pé; (C) Radiografia pós-operatória em incidência anteroposterior com o paciente em pé, 2 anos após a cirurgia; (D) Radiografia pós-operatória em incidência em perfil com o paciente em pé, 2 anos após a cirurgia. 

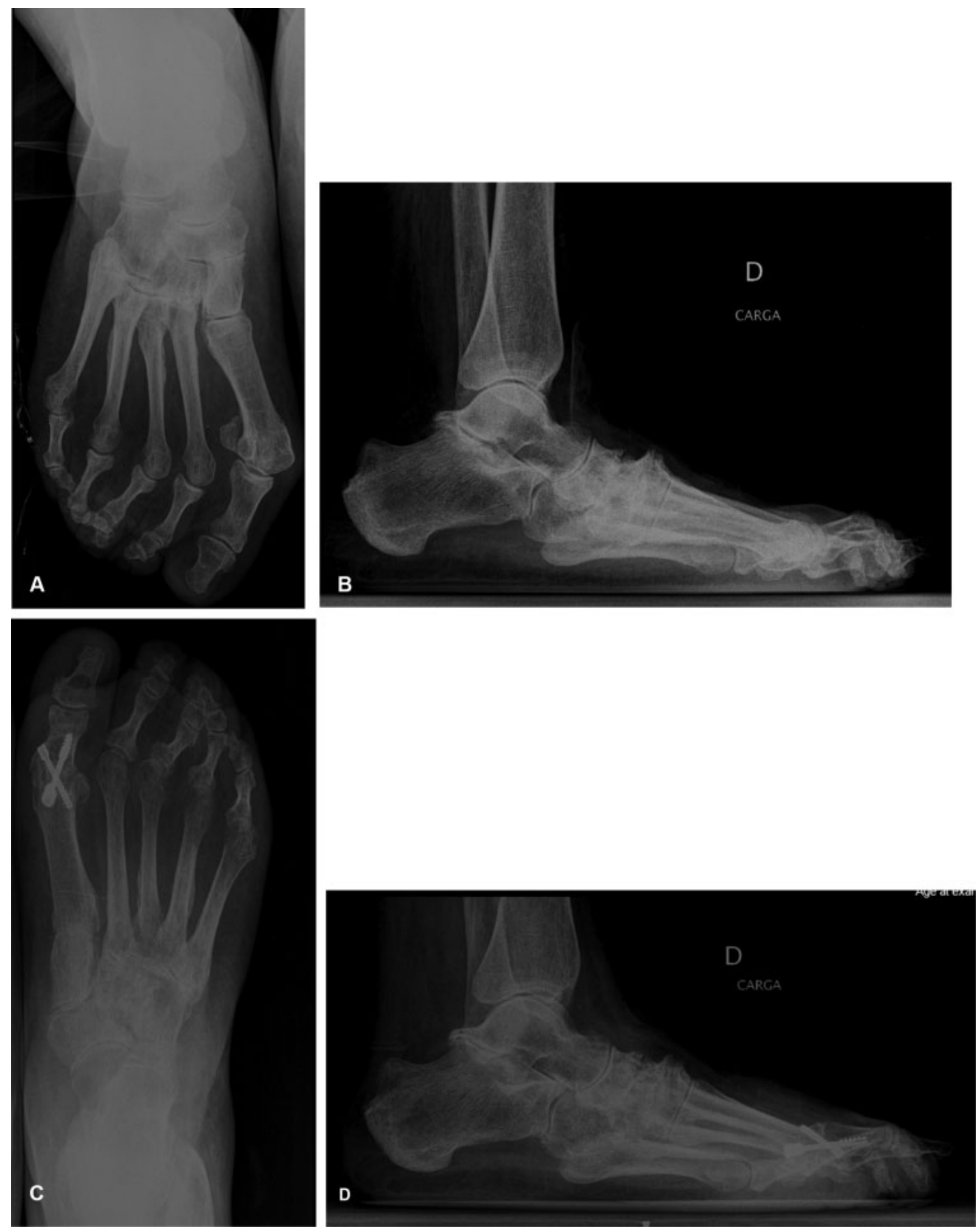

Fig. 4 Imagens radiográficas pré- e pós-operatórias de uma mulher de 58 anos de idade com hallux rigidus submetida à artroplastia de 1MTP. (A) Radiografia pré-operatória em incidência anteroposterior com a paciente em pé; (B) Radiografia pré-operatória em incidência em perfil com a paciente em pé; (C) Radiografia pós-operatória em incidência anteroposterior com a paciente em pé, 3 anos após a cirurgia; (D) Radiografia pósoperatória em incidência em perfil com a paciente em pé, 3 anos após a cirurgia. 


\section{Discussão}

O hallux rigidus afeta cerca de $10 \%$ da população e é mais prevalente em mulheres entre 60 e 70 anos de idade. ${ }^{6,18} \mathrm{~A}$ artrodese de 1MTP impede a movimentação da articulação, diminuindo a dor e estabilizando a coluna medial do pé, e ainda é o tratamento padrão-ouro para pacientes com artrite avançada. ${ }^{19-21}$ Esse procedimento, porém, não é isento de complicações. Aproximadamente $10 \%$ dos pacientes com fusão apresentam ausência de consolidação e requerem a realização de novas cirurgias; ${ }^{22,23}$ além disso, alguns pacientes se queixam continuamente da rigidez do pé e da alteração dos padrões de marcha, com redução do comprimento do passo e perda da flexão plantar do tornozelo na ponta do pé fundido. ${ }^{18}$ Isso é muito importante em pacientes com hallux rigidus bilateral, em que uma fusão bilateral altera a biomecânica normal da passada e da marcha.

A artroplastia de 1MTP pode manter a marcha e a biomecânica mais próximas da fisiologia. Embora os resultados com os primeiros implantes de MTP tenham sido decepcionantes, a melhor compreensão da biomecânica do pé permitiu o desenvolvimento de novos implantes com melhores resultados clínicos e taxas de sobrevida. Em uma metaanálise com 3.049 artroplastias de 1MTP e período médio de acompanhamento de 61 meses, a taxa de satisfação após esses procedimentos foi de $94,5 \%{ }^{12}$

No presente estudo, os dois grupos apresentaram uma melhora significativa da dor e das pontuações funcionais e clínicas, inclusive nos pacientes submetidos a artroplastia. Esses achados estão de acordo com parte da literatura mais recente sobre a artroplastia de $1 \mathrm{MTP} .^{6,18,19,24}$ Enquanto alguns estudos compararam os resultados da artrodese e da artroplastia de 1MTP, a maioria analisou próteses de gerações anteriores, associadas a piores resultados. ${ }^{12,24-28}$ Neste estudo, no entanto, os pacientes do grupo da artroplastia apresentaram melhores resultados clínicos e funcionais do que aqueles submetidos à fusão.

A taxa de complicações também foi menor no grupo de artroplastia em comparação com o grupo de artrodese (5,5\% versus $22,1 \%$ ). A ausência de consolidação foi observada em $16,6 \%$ dos pacientes submetidos à fusão, com necessidade de nova cirurgia de revisão. Essa taxa de ausência de consolidação é equivalente àquelas já relatadas em técnicas semelhantes de fusão com placas ou parafusos. ${ }^{12,19,22}$ Embora outros autores tenham relatado taxas relevantes de não consolidação após a fusão, ${ }^{29}$ nenhum caso foi observado no presente estudo. A infecção também é uma grande preocupação nos pacientes submetidos à artroplastia. Neste estudo, a única complicação no grupo da artroplastia foi uma infecção, o que está de acordo com relatos anteriores. ${ }^{24}$

Os resultados clínicos superiores aqui encontrados talvez reflitam a seleção cuidadosa de pacientes para a artroplastia. O autor acredita que os pacientes selecionados para a artroplastia não devem apresentar elevação (elevatus) do primeiro metatarso, retração do sistema calcâneo-plantar e qualquer desalinhamento do primeiro raio do pé. Atenção especial também deve ser dada ao comprimento do primeiro raio; em pacientes com index plus e para evitar sobrecarregar a artroplastia, o comprimento do primeiro metatarso deve ser reduzido e se igualar ao segundo metatarso para preservação da biomecânica normal do pé. Somente esses casos, em que havia possibilidade de adaptação estrutural e anatômica da prótese, foram submetidos às artroplastias; os demais casos foram submetidos à fusão.

Os principais pontos fortes do presente artigo são o fato de ser um estudo comparativo em que todos os pacientes foram operados pelo mesmo cirurgião que, além de utilizar uma técnica padronizada, não participou da coleta de dados e, portanto, não influenciou a análise dos dados. Além disso, o período de acompanhamento dos pacientes submetidos à artroplastia foi relativamente longo (média de 6,75 anos, máximo de 9,17 anos), sem identificação de nenhuma outra complicação apesar da avaliação clínica e radiográfica.

O presente estudo também apresenta algumas limitações, pois é de natureza retrospectiva e a amostra é relativamente pequena. Além disso, os pacientes não foram designados aleatoriamente para cada grupo e foram selecionados para serem submetidos à artrodese ou artroplastia dependendo do alinhamento do primeiro raio e das características pessoais. Como já mencionado, o autor acredita que essa seleção de pacientes é essencial para o sucesso dessa técnica.

\section{Conclusões}

Em conclusão, os dois métodos cirúrgicos utilizados tiveram resultados satisfatórios. A artroplastia de 1MTP demonstrou resultados clínicos e funcionais superiores, sendo uma boa alternativa para os candidatos adequados. No entanto, uma amostra maior e um acompanhamento mais longo são necessários à obtenção de resultados conclusivos.

Conflito de Interesses

Os autores declaram não haver conflito de interesses.

\section{Referências}

1 Coughlin MJ, Shurnas PS. Hallux rigidus: demographics, etiology, and radiographic assessment. Foot Ankle Int 2003;24(10):731-743

2 Ho B, Baumhauer J. Hallux rigidus. EFORT Open Rev 2017;2(01): $13-20$

3 Hamid KS, Parekh SG. Clinical Presentation and Management of Hallux Rigidus. Foot Ankle Clin 2015;20(03):391-399

4 Polzer H, Polzer S, Brumann M, Mutschler W, Regauer M. Hallux rigidus: Joint preserving alternatives to arthrodesis - a review of the literature. World J Orthop 2014;5(01):6-13

5 Coughlin MJ, Shurnas PS. Hallux rigidus. Grading and long-term results of operative treatment. J Bone Joint Surg Am 2003;85(11): 2072-2088

6 Dülgeroğlu TC, Metineren H. Treatment of End-Stage Hallux Rigidus Using Total Joint Arthroplasty: A Short-Term Clinical Study. J Foot Ankle Surg 2017;56(05):1047-1051

7 Beertema W, Draijer WF, van Os JJ, Pilot P. A retrospective analysis of surgical treatment in patients with symptomatic hallux rigidus: long-term follow-up. J Foot Ankle Surg 2006;45(04): 244-251

8 DeFrino PF, Brodsky JW, Pollo FE, Crenshaw SJ, Beischer AD. First metatarsophalangeal arthrodesis: a clinical, pedobarographic and gait analysis study. Foot Ankle Int 2002;23(06):496-502

9 Konkel KF, Menger AG, Retzlaff SA. Mid-term results of Futura hemi-great toe implants. Foot Ankle Int 2008;29(08):831-837 
10 Swanson AB, Lumsden RM, Swanson GD. Silicone implant arthroplasty of the great toe. A review of single stem and flexible hinge implants. Clin Orthop Relat Res 1979;(142):30-43

11 Brewster M. Does total joint replacement or arthrodesis of the first metatarsophalangeal joint yield better functional results? A systematic review of the literature. J Foot Ankle Surg 2010;49 (06):546-552

12 Cook E, Cook J, Rosenblum B, Landsman A, Giurini J, Basile P. Metaanalysis of first metatarsophalangeal joint implant arthroplasty. J Foot Ankle Surg 2009;48(02):180-190

13 Sullivan MR. Hallux rigidus: MTP implant arthroplasty. Foot Ankle Clin 2009;14(01):33-42

14 Dening J, van Erve RH. Arthrodesis of the first metatarsophalangeal joint: a retrospective analysis of plate versus screw fixation. J Foot Ankle Surg 2012;51(02):172-175

15 Foote KM, Teasdall RD, Tanaka ML, Scott AT. First metatarsophalangeal arthrodesis: a biomechanical comparison of three fixation constructs. J Surg Orthop Adv 2012;21(04):223-231

16 Mohammed R, Gadgil A. Molded arthrodesis of the hallux metatarsophalangeal joint using the crossed-screw technique: surgical technique, results and functional outcomes. Foot Ankle Surg 2012; 18(02):132-135

17 Silva LF, Sousa CV, Rodrigues Pinto R, Santos C, Geada JM. Preliminary Results from the Metis-Newdeal((R)) Total Metatarsophalangeal Prosthesis. Rev Bras Ortop 2015;46(02):200-204

18 Baumhauer JF, Singh D, Glazebrook M, Blundell C, De Vries G, Le IL, Nielsen D, Pedersen ME, Sakellariou A, Solan M, Wansbrough G, Younger AS, Daniels T; for and on behalf of the CARTIVA Motion Study Group. Prospective, Randomized, Multi-centered Clinical Trial Assessing Safety and Efficacy of a Synthetic Cartilage Implant Versus First Metatarsophalangeal Arthrodesis in Advanced Hallux Rigidus. Foot Ankle Int 2016;37(05):457-469

19 Donegan RJ, Blume PA. Functional Results and Patient Satisfaction of First Metatarsophalangeal Joint Arthrodesis Using Dual Crossed Screw Fixation. J Foot Ankle Surg 2017;56(02):291-297
20 Horisberger M, Haeni D, Henninger HB, Valderrabano V, Barg A. Total Arthroplasty of the Metatarsophalangeal Joint of the Hallux. Foot Ankle Int 2016;37(07):755-765

21 Karlock LG, Berry L, Craft ST, Petrozzi R, Grahn AG, Casteel ML. First Metatarsophalangeal Joint Fusion With Use of Crossed Kirschner Wires and Intramedullary Steinmann Pin. J Foot Ankle Surg 2017;56(06):1139-1142

22 Mirmiran R, Wilde B, Nielsen M. Retrospective analysis of the rate and interval to union for joint arthrodesis of the foot and ankle. J Foot Ankle Surg 2014;53(04):420-425

23 Raikin SM, Ahmad J, Pour AE, Abidi N. Comparison of arthrodesis and metallic hemiarthroplasty of the hallux metatarsophalangeal joint. J Bone Joint Surg Am 2007;89(09):1979-1985

24 Erkocak OF, Senaran H, Altan E, Aydin BK, Acar MA. Short-term functional outcomes of first metatarsophalangeal total joint replacement for hallux rigidus. Foot Ankle Int 2013;34(11): 1569-1579

25 Gibson JN, Thomson CE. Arthrodesis or total replacement arthroplasty for hallux rigidus: a randomized controlled trial. Foot Ankle Int 2005;26(09):680-690

26 Pulavarti RS, McVie JL, Tulloch CJ. First metatarsophalangeal joint replacement using the bio-action great toe implant: intermediate results. Foot Ankle Int 2005;26(12):1033-1037

27 Stevens J, de Bot RTAL, Hermus JPS, van Rhijn LW, Witlox AM. Clinical Outcome Following Total Joint Replacement and Arthrodesis for Hallux Rigidus: A Systematic Review. JBJS Rev 2017;5 (11):e2

28 Stone OD, Ray R, Thomson CE, Gibson JN. Long-Term Follow-up of Arthrodesis vs Total Joint Arthroplasty for Hallux Rigidus. Foot Ankle Int 2017;38(04):375-380

29 Kim PJ, Hatch D, Didomenico LA, Lee MS, Kaczander B, Count G, Kravette M. A multicenter retrospective review of outcomes for arthrodesis, hemi-metallic joint implant, and resectional arthroplasty in the surgical treatment of end-stage hallux rigidus. J Foot Ankle Surg 2012;51(01):50-56 